\title{
Psychological consequences of COVID-19 pandemic in Italian MS patients: signs of resilience?
}

\author{
Rocco Capuano ${ }^{1} \cdot$ Manuela Altieri $^{1,2} \cdot$ Alvino Bisecco $^{1} \cdot$ Alessandro d'Ambrosio $^{1} \cdot$ Renato Docimo $^{1}$. \\ Daniela Buonanno ${ }^{1}$. Federica Matrone ${ }^{1} \cdot$ Federica Giuliano $^{1} \cdot$ Gioacchino Tedeschi ${ }^{1} \cdot$ Gabriella Santangelo $^{2}$. \\ Antonio Gallo ${ }^{1}$
}

Received: 27 May 2020 / Revised: 17 July 2020 / Accepted: 20 July 2020 / Published online: 28 July 2020

(c) The Author(s) 2020

\begin{abstract}
Background Anxiety, depression and reduction of quality of life (QoL) are common in people with multiple sclerosis (pwMS). Fear of getting sick from COVID-19, government's lockdown and the imposed social distancing might have had an impact on psychological distress and QoL.

Objectives The aim of our study was to investigate anxiety, depression and QoL changes in pwMS during SARS-CoV-2 outbreak and lockdown in Italy.

Methods 67 pwMS with a previous (less than 6 months) neuropsychological evaluation before SARS-CoV-2 outbreak (T0) were re-evaluated at the time of the outbreak and lockdown in Italy (T1). They underwent a clinical and neurological evaluation and completed the State-Trait Anxiety Inventory (STAI-Y1), the Beck Depression Inventory second edition (BDI-II), and Multiple Sclerosis Quality of Life-54 (MsQoL-54) at T0 and T1. Benjamini-Hochberg procedure was applied to control the false discovery rate.

Results BDI-II and STAI-Y1 scores did not change between T0 and T1. At T1, MsQoL-54 scores were higher on the satisfaction with sexual life and the social function subscales, and lower on the limitation due to emotional problems subscale.

Conclusions This is the first study that evaluated mood and QoL levels before and during the lockdown due to COVID-19 pandemic in pwMS. No worsening of anxiety and depression levels was found. Contrariwise some improvements were noted on QoL, the most reliable regarding the sexual satisfaction and the social function.
\end{abstract}

Keywords COVID-19 $\cdot$ Lockdown $\cdot$ Multiple sclerosis $\cdot$ Anxiety $\cdot$ Depression $\cdot$ Sexual satisfaction

\section{Introduction}

Between the end of February and the beginning of March 2020, SARS-CoV-2, the causative agent of COVID-19, quickly spread around the world, endangering the health of people, especially those with older age and/or chronic illnesses [1]. Italy was strongly hit by COVID-19 pandemic, therefore the Italian Government decreed urgent measures

Rocco Capuano and Manuela Altieri share the first authorship.

Antonio Gallo

antonio.gallo@unicampania.it

1 Department of Advanced Medical and Surgical Sciences, University of Campania “Luigi Vanvitelli”, Naples, Italy

2 Department of Psychology, University of Campania "Luigi Vanvitelli", Caserta, Italy promoting social distancing to limit the spread of the virus. In fact, since March 11th, all not indispensable work, social, sporting, retail and recreational activities were suspended or, where possible, converted to the so-called smart-working. Certainly, promoting social distancing is essential to prevent the spreading of SARS-CoV-2 and to ensure public health; on the other hand, changing lifestyle-drastically limiting working and social activities - together with uncertainty and health concerns, might have a significant detrimental effect on mood and mental health.

Multiple Sclerosis (MS) patients, particularly those treated with immunosuppressive drugs, high disability and long-lasting disease might be more susceptible to COVID19 and its complications, therefore new statement on MS management during SARS-CoV-2 pandemic has been drawn up [2, 3]. Furthermore, mood disorders, particularly anxiety and depression, are common in people with 
MS (pwMS). Rates of anxiety and depression are higher in pwMS compared with age-matched healthy individuals and patients with other chronic illnesses [4]. Depending on method and study population, prevalence rates for anxiety varies between 20 and $40 \%$ [5-8], while the prevalence of depression ranges between 14 and 50\% [5, 9-11] with a lifetime prevalence of $50 \%[11,12]$.

Giordano and colleagues reported that, in Italy, $43 \%$ of pwMS suffers from anxiety and 34\% from depression [13]. Several factors can help explaining the higher prevalence of anxiety and depression in pwMS: (1) the natural reaction to the unpredictable course of the disease, (2) the need to be constantly treated with disease modifying as well as symptomatic drugs, (3) the psychosocial impact of MS in life goals, employment, relationships, and activities of daily living, and (4) disease-related brain structural and functional changes [4, 14-16].

Social and working restrictions imposed by the lockdown as well as the fear of getting severely sick might have influenced anxiety and depression levels of pwMS. The aim of our study was, therefore, to investigate-in a group of pwMS in which a pre-pandemic neuropsychological and behavioral evaluation was available-changes in lifestyle, levels of anxiety, depression and quality of life (QoL) during the Italian lockdown due to SARS-CoV-2 pandemic.

\section{Methods}

Seventy-five relapsing-remitting (RR) MS patients that had a pre-lockdown (T0) neurological, neuropsychological and behavioral evaluation between September and December 2019 as part of the ongoing clinical and/or research activities at the MS Center of the I Division of Neurology of the University of Campania "Luigi Vanvitelli" were selected. Patients were reached at the phone during the lockdown between April 16th and April the 23rd, 2020, when they were invited to participate to a second psychological and behavioral assessment (T1). To be included in the study, patients had to have a diagnosis of RRMS according to last revised McDonald criteria [17]; they had to be relapse- and steroid-free within the month prior to baseline assessment (T0) and during the time-window between baseline and follow-up assessment (T0-T1). The study was approved by the local Ethical Committee. All participants were informed on the objectives of the study and provided informed consent by means of an online form.

\section{Clinical assessment}

At T0, the following data were recorded from each participating subject: the Expanded Disability Status Scale [18] score, the disease duration, and the disease modifying therapy (DMT). DMTs were classified as low/ moderate-efficacy DMTs (dimethyl fumarate, glatiramer acetate, interferons, teriflunomide) and moderate/highefficacy DMTs (alemtuzumab, fingolimod, natalizumab, ocrelizumab).

At T1, we conducted structured telephone calls to enrol patients and, after receiving informed consent, a clinical and neurological assessment evaluating for COVID-19 symptoms. Occurence of relapses and/or DMTs changes, was performed via telephone call by a neurologist with expertise in MS.

\section{Neuropsychological and behavioral assessment}

The following neuropsychological tests and behavioral scales were administered at T0: (1) the Symbol Digit Modalities Test (SDMT) a neuropsychological test that evaluates processing speed efficiency and represents a valuable surrogate marker of global cognitive status [19, 20]. For the purposes of the study, raw scores of SDMT were converted into corrected scores according to the formula provided by Italian normative data [19] and then they were transformed into z-scores. pwMS were considered cognitively impaired if their SDMT z-scores were $\leq 1.5$ standard deviations; (2) the State Anxiety section of the Italian version of the State-Trait Anxiety Inventory (STAIY-1) [21] to evaluate state-anxiety levels; STAI-Y-1 scores range from 0 to 80 and higher scores are indicative of higher levels of state-anxiety; (3) the Italian version of the Beck Depression Inventory-II Edition (BDI-II) [22] to investigate patients' depressive symptomatology; the scores range from 0 to 63 and scores higher than 18 are indicative of depressive symptomatology; (4) the Italian version of the Multiple Sclerosis Quality of Life-54 (MSQoL-54) [23] to estimate the QoL. MSQoL-54 provides a number of indices to evaluate different aspects of QoL: physical health, limitations due to physical and emotional problems, pain, emotional well-being, energy, health perceptions, social, cognitive and sexual functions, health distress, change in health, satisfaction with sexual function, overall quality of life. Moreover, composite scores related to physical (PHCs) and mental (MHCs) well-being are provided. The scores of each subscale range from 0 to 100 (lower and higher levels of QoL, respectively).

At T1, a trained neuropsychologist, who tested patients at $\mathrm{T} 0$, proceeded by sending an email with a link to an 
online form. The structured self-assessment consisted of: (1) a survey aimed to investigate lifestyle changes and feelings during the Italian lockdown; out of 20 items listed in the survey, the most relevant explored/regarded: fear of getting COVID-19 having MS and/or being treated with DMTs; change in adherence to DMTs; repercussions on working habits; changes of social habits; self-perception of changes; (2) the Italian version of STAI-Y-1; (3) the Italian version of BDI-II; (4) the Italian male and females versions of MSQoL-54.

\section{Statistical analyses}

All analyses were performed using SPSS (SPSS Statistics version 25.0).

Absolute numbers and percentage were used to describe categorical variables, means and standard deviations (SD) or medians and interquartile ranges (P25-P75) were used for continuous variables.

STAI-Y-1, BDI-II and MSQoL-54 scores at T0 and T1 were compared using the paired $t$ test ( $t$ test for dependent samples) or chi-square where appropriate. A subgroup analysis was performed to evaluate possible differences on depression, state-anxiety and QoL, at T0 and T1, in males and females. Correlations between significant $t$ test variables and BDI-II, STAI-Y1, MSQoL-54 subscales, including PHCs and MHCs at T0 and T1 were evaluated by Pearson correlation coefficient.

Three sensitivity analyses were performed by excluding: (1) baseline depressed patient; (2) baseline anxious patients; (3) baseline cognitive-impaired patients. A $p$ value of 0.05 was the cut-off for significance. Benjamini-Hochberg procedure was applied to control the false discovery rate [24].

\section{Results}

Sixty-seven out of 75 patients with the T0 evaluation agreed to participate and were enrolled in the study. Two patients declined to participate, while six were not reachable at the time of the survey. Patients' socio-demographic and clinical characteristics registered at $\mathrm{T} 0$ are summarized in Table 1. No participants changed their DMT between the two timepoints. Six out of 67 patients (9\%) reported symptoms possibly due to SARS-CoV-2 infection, but only 1 (1.5\%) underwent a double negative nasopharyngeal swab for diagnosis of COVID-19; any patient nor their family members or cohabitants were diagnosed with COVID-19. Thirty-eight (56.7\%) patients reported to feel at higher risk of getting COVID-19 due to MS, while 30 (44.8\%) patients reported that the cause of being more at risk of getting COVID-19 was related to being on a DMT. However, the vast majority of the patients $(N=66 ; 98.5 \%)$ did not report any change in
Table 1 Socio-demographic and clinical characteristics of Multiple Sclerosis sample before COVID-19 lockdown $(\mathrm{N}=67)$

\begin{tabular}{ll}
\hline Mean Age (years, SD) & $37.5(11.1)$ \\
Sex (M/F) & $30 / 37$ \\
Mean years of education (SD) & $13.7(3.5)$ \\
Mean disease duration (months, SD) & $91.5(96.9)$ \\
Median EDSS (P25-P75) & $2.0(1.0-2.0)$ \\
Mean SDMT (SD) & $49.9(13.4)$ \\
DMTs, $n(\%)$ & \\
None & $8(11.9)$ \\
Low/moderate efficacy & $39(58.2)$ \\
Moderate/high efficacy & $20(29.9)$ \\
\hline
\end{tabular}

$S D$ standard deviation, EDSS Expanded Disability Status Scores, P25 25th percentile, $P 75$ 75th percentile, SDMT Symbol Digit Modalities Test, DMTs disease modifying therapies

adherence to DMT schedule; only one patient reduced the adherence but did not stop DMT assumption. Thirty-four (50.7\%) patients reported that they contacted a physician to get more information about COVID-19 (6\% contacted their family doctor; $34.3 \%$ contacted doctors of their MS Center; $10.4 \%$ contacted both).

Fifty-seven $(85.1 \%)(N=57)$ patients reported significant changes in social and lifestyle habits, while 39 (58.3\%) reported more difficulties in daily life. Twenty out of 67 patients $(29.8 \%)$ were unemployed at the time of SARSCoV-2 outbreak. Only 7 patients (10.5\%) continued to work in the same modality as before the lockdown, while 16 (23.9\%) worked from home (smart-working) and 24 (35.8\%) stopped working at all.

As regards the mood and behavioral status of the patients, 8 patients (11.9\%) were depressed and $8(11.9 \%)$ were anxious at $\mathrm{T} 0$; at $\mathrm{T} 1$, the same prevalence of depression was found, while 11 patients (16.4\%) reported state-anxiety. However, no significant increase in anxiety prevalence was observed during the lockdown $\left(16.4 \%, \chi^{2}=0.488\right.$, $p=0.485)$. No differences were found on BDI-II and STAIY1 scores between $\mathrm{T} 0$ and $\mathrm{T} 1$ (Table 2).

After correction for multiple comparisons, at $\mathrm{T} 1$ the scores of the following subscales of the MsQoL-54 showed significant changes: higher scores on the satisfaction with sexual function subscale $(p<0.001)$, lower scores on the limitations due to emotional problems subscale $(p=0.003)$ and higher scores on the subscales addressing social function $(p=0.003)$.

At T1, a trend was found on changes in perceived health status $(p=0.025)$, but it did not survive the correction for multiple comparison. Patients were asked to explain reasons of improvement and $58.7 \%$ attributed the amelioration to increased partner support, $30.4 \%$ to reduced workrelated stress and $10.9 \%$ was not able to specify the reason. Moreover, the satisfaction with sexual function subscale 
Table 2 Behavioral and quality of life assessment pre- and during the COVID-19 lockdown in Italy

\begin{tabular}{|c|c|c|c|c|}
\hline & T0 & T1 & $T$ & $p$ \\
\hline BDI-II-mean (SD) & $9.94(7.9)$ & $8.6(9.3)$ & 1.589 & .117 \\
\hline STAI-Y1-mean (SD) & $42.1(11.4)$ & $43.5(12)$ & -1.005 & .319 \\
\hline MSQoL physical health—mean (SD) & $78.5(26.9)$ & $82.6(24.4)$ & -1.716 & .091 \\
\hline MSQoL limitations due to physical problems-mean (SD) & $77.4(35.2)$ & $77.3(35.2)$ & .015 & .988 \\
\hline MSQoL limitations due to emotional problems-mean (SD) & $81.5(32.7)$ & $67.7(40.7)$ & 3.102 & .003 \\
\hline MSQoL pain-mean (SD) & $75.4(25.3)$ & $82(25)$ & -1.876 & .065 \\
\hline MSQoL emotional well-being-mean (SD) & $64.2(18.4)$ & $64.3(21.4)$ & -.059 & .954 \\
\hline MSQoL energy-mean (SD) & $51.1(18.2)$ & $48.1(17.9)$ & 1.518 & .134 \\
\hline MSQoL health perceptions-mean (SD) & $54.4(20.9)$ & $55.9(21.4)$ & -.705 & .483 \\
\hline MSQoL social function-mean (SD) & $77.7(17.4)$ & $84.2(20.4)$ & -3.133 & .003 \\
\hline MSQoL cognitive function-mean (SD) & $72.7(18.2)$ & $74.3(23.6)$ & -.742 & .461 \\
\hline MSQoL health distress-mean (SD) & $72.9(22.4)$ & $74.2(23.9)$ & -.621 & .537 \\
\hline MSQoL sexual function-mean (SD) & $83.3(26)$ & $87.2(21.8)$ & -1.066 & .290 \\
\hline MSQoL change in health-mean (SD) & $44.9(22.3)$ & $53.5(21.8)$ & -2.295 & .025 \\
\hline MSQoL satisfaction with sexual function—mean (SD) & $70.1(25.7)$ & $89.3(21.1)$ & -5.791 & $<.001$ \\
\hline MSQoL overall quality of life—mean (SD) & $64.2(21.3)$ & $68.4(16.3)$ & -1.702 & .094 \\
\hline MSQoL physical health composite score-mean (SD) & $70.2(17.8)$ & $72.9(19.8)$ & -1.487 & .142 \\
\hline MSQoL mental health composite score-mean (SD) & $71.1(17.7)$ & $68.7(21.8)$ & 1.278 & .206 \\
\hline
\end{tabular}

Values at $\mathrm{T} 0$ and $\mathrm{T} 1$ are expressed as mean (standard deviation). TO before the lockdown, $T 1$ during the lockdown, $t$ paired sample $t$ test, $p$ probability value, BDI-II Beck Depression Inventory, second edition, STAI-State State Anxiety section of the State-Trait Anxiety Inventory (STAI-Y-1), MSQoL-54 Multiple Sclerosis Quality of Life-54. Significant values $(p<.05)$ are reported in bold. Significant values after Benjamini-Hochberg procedure are underlined was significantly and negatively correlated with BDI-II and STAI-Y1 scores and positively with both PHCs and MHCs, at T0 (BDI-II: $r=-0.397, p=0.001$; STAI-Y1: $r=-0.261$, $p=0.041$; PHCs: $r=0.528, p<0.001$; MHCs: $r=0.482$, $p \leq 0.001$ ) and T1 (BDI-II: $r=-0.472, p<0.001$; STAI-Y1: $r=-0.437, p<0.001$; PHCs: $r=0.543, p<0.001$; MHCs: $r=0.579, p \leq 0.001)$.

Moving on sex differences (Table 3), we found that women scored higher than men on BDI-II $(t=-2.833$, $p=0.006)$ and STAI-Y1 $(-3.121, p=0.003)$ at T0, but not at T1 (BDI-II: $t=-0.869, p=0.388$; STAI-Y1: $t=-1.176$, $p=0.244$ ). The intragroup analysis (Table 3 ) to evaluate possible differences between $\mathrm{T} 0$ and $\mathrm{T} 1$ revealed some trends that lost significance after Benjamini-Hochberg correction: higher STAI-Y1 scores in males at T1 $(t=-2.105$; $p=0.044)$ and lower BDI-II scores in females at T1 $(t=2.162 ; p=0.037)$. Moreover, trends between T0 and T1 were found on the following MSQoL-54 subscales (Table 3): pain subscale ( $t=-3.191, p=0.004)$ in males; social function subscale and satisfaction with sexual function in both males $(t=-2.465, p=0.02 ; t=-2.491, p=0.019$, respectively) and females ( $t=-2.062, p=0.047 ; t=-6.068$, $p<0.001)$; limitations due to emotional problems subscale and overall quality of life $(t=2.443, p=0.020 ; t=-3.163$, $p=0.003$, respectively) in females. After applying the Benjamini-Hochberg procedure, only the differences between $\mathrm{T} 0$ and $\mathrm{T} 1$ on the satisfaction with sexual function and overall
QoL subscales of MSQoL-54 in females remained significant. Lastly, three sensitivity analysis were performed by excluding, respectively, anxious, depressed and cognitively impaired patients at $\mathrm{T} 0$ and no differences were found with respect to the whole sample.

\section{Discussion}

In the present study, we investigated lifestyle changes together with levels of anxiety, depression and QoL in pwMS during the Italian lockdown due to SARS-CoV-2 pandemic. Notably, we designed the study to enrol only pwMS for whom a recent neuropsychological and behavioural assessment was already available before the COVID19 lockdown. By designing the study in this way, we were able to have a baseline/reference time-point against which to compare the scores measured during the lockdown. To the best of our knowledge, only anxiety was investigated in a small cross-sectional study conducted on Iranian pwMS during SARS-CoV-2 outbreak [25].

As expected, several patients significantly changed their social and lifestyle habits with more difficulties encountered in daily life due to the country lockdown. More than a half of the patients had to change their work habits by stopping working or starting smart-working, while only the $10.5 \%$ of 
Table 3 Behavioral and quality of life (QoL) assessment pre- and during the COVID-19 lockdown in Italy in females and males pwMS

\begin{tabular}{|c|c|c|c|c|c|c|c|c|}
\hline & \multicolumn{4}{|l|}{ FEMALES } & \multicolumn{4}{|l|}{ MALES } \\
\hline & T0 & $\mathrm{T} 1$ & $t$ & $p$ & T0 & T1 & $t$ & $p$ \\
\hline BDI-II—mean (SD) & $12.3(9)$ & $9.5(10.9)$ & 2.162 & .037 & $7.03(5.2)$ & $7.5(7.40)$ & -.510 & .614 \\
\hline STAI-Y1-mean (SD) & $45.81(10.8)$ & $45(12.1)$ & .396 & .694 & $37.5(10.7)$ & $41.6(11.76)$ & -2.105 & .044 \\
\hline MSQoL-54 physical health—mean (SD) & $78.3(26.9)$ & $80.7(28.0)$ & -.727 & .472 & $78.7(27.5)$ & $85.0(19.10)$ & -1.803 & .083 \\
\hline $\begin{array}{l}\text { MSQoL-54 limitations due to physical problems-mean } \\
\text { (SD) }\end{array}$ & $80(34.1)$ & $77.1(35.5)$ & .399 & 693 & $74.1(36.9)$ & $77.7(35.57)$ & -.518 & .608 \\
\hline $\begin{array}{l}\text { MSQoL-54 limitations due to emotional problems- } \\
\text { mean (SD) }\end{array}$ & $81.9(31.8)$ & $67.6(41.8)$ & 2.443 & .020 & $80.9(34.5)$ & $67.9(40.04)$ & 1.890 & .070 \\
\hline MSQoL-54 pain-mean (SD) & $76.2(25.9)$ & $77.1(29.4)$ & -.171 & .865 & $74.3(24.8)$ & $88.4(16.11)$ & -3.191 & .004 \\
\hline MSQoL-54 emotional well-being-mean (SD) & $59.6(17.7)$ & $60.1(22.8)$ & -.290 & .835 & $70.1(17.8)$ & $69.7(18.55)$ & .121 & .905 \\
\hline MSQoL-54 energy—mean (SD) & $48.1(17.7)$ & $44.6(17.4)$ & 1.469 & .151 & $55(18.3)$ & $52.7(17.69)$ & .688 & .497 \\
\hline MSQoL-54 health perceptions-mean (SD) & $52.6(19.8)$ & $53.7(22.4)$ & -.363 & .719 & $56.8(22.3)$ & $58.6(19.99)$ & -.757 & .456 \\
\hline MSQoL-54 social function-mean (SD) & $76.6(17.6)$ & $82.9(23.6)$ & -2.062 & .047 & $79.2(17.3)$ & $86.0(15.56)$ & -2.465 & .020 \\
\hline MSQoL-54 cognitive function-mean (SD) & $69.7(19)$ & $73.9(24.1)$ & -1.574 & .124 & $76.6(16.7)$ & $74.8(23.27)$ & .532 & .599 \\
\hline MSQoL health distress-mean (SD) & $69.7(23.7)$ & $70.3(26.4)$ & -.226 & .823 & $76.9(20.5)$ & $79.3(19.61)$ & .615 & .544 \\
\hline MSQoL sexual function-mean (SD) & $80(29)$ & $87.9(22.9)$ & -1.554 & .129 & $87.5(21.6)$ & $86.3(20.69)$ & .239 & .813 \\
\hline MSQoL change in health-mean (SD) & $46.5(22.5)$ & $54.9(20.5)$ & -1.784 & .083 & $42.9(22.4)$ & $51.8(23.50)$ & -1.441 & .161 \\
\hline MSQoL satisfaction with sexual function-mean (SD) & $68.2(23.7)$ & $92.4(15.9)$ & -6.068 & $<.001$ & $72.3(28.3)$ & $85.7(25.84)$ & -2.491 & .091 \\
\hline MSQoL overall quality of life—mean (SD) & $59.5(20)$ & $69.2(15.5)$ & -3.163 & .003 & $70.2(21.7)$ & $67.4(17.61)$ & .716 & .480 \\
\hline MSQoL physical health composite score-mean (SD) & $68.8(17.4)$ & $70.7(21.5)$ & -.742 & .463 & $72(18.5)$ & $75.7(17.39)$ & -1.430 & .164 \\
\hline MSQoL mental health composite score-mean (SD) & $68.5(15.9)$ & $67(22.8)$ & 680 & .501 & 74.4(19.5) & $70.9(20.65)$ & 1.097 & .283 \\
\hline
\end{tabular}

Values at T0 and T1 are expressed as mean (standard deviation). T0 before the lockdown, $T 1$ during the lockdown, $t$ paired sample $t$ test, $p$ probability value, BDI-II Beck Depression Inventory, second edition, STAI-Y1 State Anxiety section of the State-Trait Anxiety Inventory, MSQoL-54 Multiple Sclerosis Quality of Life-54. Significant values $(p<0.05)$ are reported in bold. Significant values after Benjamini-Hochberg procedure are underlined

subjects continued working in the same modalities as before the lockdown.

Despite adaptation to home restrictions, no differences in levels of anxiety or depression were found between $\mathrm{T} 1$ and T0. These results are in apparent contrast with a recent Italian survey conducted on the general population (age range 18-90 years), which reported a high psychological distress during the lockdown [26]. This difference, indeed, might be explained by one or more of the following: (1) our sample was mostly constituted by young adults with MS; even if all people are at risk of psychological harm when kept in "isolation", children, adolescents and older adults are the most vulnerable while young adults are the most resilient [27]; (2) since the studied population was from Campania, an Italian region that was affected by the pandemic much less than other northern regions [28], it is possible that levels of fear of getting COVID-19 were lower; (3) pwMS might be more accustomed to live with higher levels of anxiety and depression compared to healthy people, showing a higher resilience to external events concerning all.

As already mentioned, the only preliminary report that investigated anxiety during COVID-19 pandemic was conducted on 33 Iranian pwMS and found high levels of anxiety [25], although the study design did not establish a pre-post comparison, therefore it was not possible to verify if levels of anxiety were already high before the lockdown. On these bases, we should have expected a significant negative impact of the pandemic in our cohort. Despite this, probably due to different study design with a T0/T1 evaluation and a larger cohort studied, we provided evidence that SARS-CoV-2 outbreak did not have a negative impact on anxiety, depression and even improved some aspects of QoL.

Moreover, we have even been able to demonstrate that, during the lockdown, pwMS reported a higher sexual satisfaction, both in the whole and in the females' group. Since we did not find differences on practical/physiological function between T0 and T1 (assessed by means of the sexual function subscale of MSQoL-54), our results strongly support the psychological component in determining the observed improvement. Sexual satisfaction is strongly related to QoL and may have a positive impact on anxiety and depression $[29,30]$. This might also help explaining the observation of a trend toward an improvement of depressive symptoms during the lockdown in female patients. Moreover, men showed a trend of higher state-anxiety levels at $\mathrm{T} 1$; this may be due to either a non-significant improvement in sexual satisfaction subscale or to higher females levels 
of anxiety at T0 [21], limiting them to further worse their anxiety within a short time frame.

In addition, we found that our cohort reported a higher social functioning. According to our survey, being at home and being more able to spend time and interact with their partners (58 out of 67 of our patients lived with a partner) and family members may have led to a higher perceived social support, having a positive impact on sexual satisfaction and mood, in line with previous studies [31-34]. Furthermore, several pwMS experience frustration, anger and other negative emotions because, compared to their healthy peers, they have more limitations in engaging in outdoor activities such as shopping, driving, participating at social and sporting activities [35]. Consequently, we can speculate that, even if they perceived themselves as more limited in daily activities, the lockdown might have been perceived by pwMS as a sort of levelling, because social restrictions caused by COVID-19 lockdown interested all people, regardless of their health status. Moreover, feelings of uncertainty about the future, fear about their own health or anxiety/frustration/anger during pandemic may be more frequent and socially accepted than before [36].

Sensitivity analysis excluding anxious, depressed or cognitive impaired patients at baseline did not change the results and this is a strength of our study.

On the other hand, this study is not without limitations. First, participants are all from Southern Italy. Even if the government decree that enacted the lockdown applied to the whole country, the spreading of COVID-19, as already said, was much slower in the Southern regions than in Northern ones; this might have an impact on the generalizability of our results. Moreover, we found lower percentages of depressed pwMS with respect to other studies [5, 9-11, 13], this result might be due to: (1) the RR phenotype and to the low levels of disability of our patients (median EDSS $=2.0$ ), in fact BDI-II scores is known to correlate with disability and MS disease course [37]; (2) the cut-off score of 18.5, which, although validated in the Italian MS population [22], is higher than other international BDI-II cut-offs [37]. Therefore, our results must be taken with caution in patients with higher levels of disability and/or depressive symptomatology. Another limitation is the absence of a comparator group of healthy subjects. Finally, even if behavioural assessments at both time points were completed with no assistance, modalities of administration differed slightly. At T0, patients completed questionnaires at our MS Center, whereas at T1, they completed questionnaires online at their own homes during the lockdown due to the Hospital rules of that period, which did not permit patients the access at our MS Centre for non-urgent conditions. Different assessment conditions may have affected final assessment result. Finally, sexual satisfaction was evaluated just based on a single/specific item of the MSQoL-54. This preliminary finding could be further explored in future studies.

In conclusion, we provided evidence that despite the tight Italian lockdown due to the COVID-19 pandemic and the fear of getting sick, we did not observe a relevant negative impact on anxiety, depression and QoL of our sample of pwMS. Contrariwise, we were even able to detect some positive effects on specific aspects of QoL that might be also interpreted as signs of resilience.

Acknowledgements Open access funding provided by Università degli Studi della Campania Luigi Vanvitelli within the CRUI-CARE Agreement. The authors thank all pwMS of our sample for the time and effort devoted to this study, and Antonia D'Agostino, Daniela Formisano, Flavia Maria Longo and Valentina Rippa for support in the scoring of the questionnaires.

Funding The authors received no specific funding for this work.

Data availability The data that support the findings of this study are available from the corresponding author, prof. Antonio Gallo, upon reasonable request.

Code availability Not applicable.

\section{Compliance with ethical standards}

Conflicts of interest RC, MA, AdA, RD, FM, FG, DB and GS have no disclosures. $\mathrm{AB}$ has received speaker honoraria and/or compensation for consulting service from Biogen, Merck and Genzyme. GT has received compensation for consulting services and/or speaking activities from Biogen, Novartis, Merck, Genzyme, Roche, Teva; and receives research support from Biogen Idec, Merck Serono, and Fondazione Italiana Sclerosi Multipla. AG received honoraria for speaking and travel grants from Merck, Genzyme, Teva, Mylan, Roche and Novartis.

Ethics approval This study was approved by the by the local Ethic Committee. All procedures performed in this study involving human participants were in accordance with the 1964 Helsinki Declaration and its later amendments or comparable ethical standards.

Consent to participate Informed consent was obtained from all individual participants included in the study.

Consent for publication The Corresponding Author transfers to Springer the publication rights and he warrants that his contribution is original and that he has full power to make this grant.

Open Access This article is licensed under a Creative Commons Attribution 4.0 International License, which permits use, sharing, adaptation, distribution and reproduction in any medium or format, as long as you give appropriate credit to the original author(s) and the source, provide a link to the Creative Commons licence, and indicate if changes were made. The images or other third party material in this article are included in the article's Creative Commons licence, unless indicated otherwise in a credit line to the material. If material is not included in the article's Creative Commons licence and your intended use is not permitted by statutory regulation or exceeds the permitted use, you will need to obtain permission directly from the copyright holder. To view a copy of this licence, visit http://creativecommons.org/licenses/by/4.0/. 


\section{References}

1. Wu C, Chen X, Cai Y et al (2020) Risk factors associated with acute respiratory distress syndrome and death in patients with coronavirus disease 2019 Pneumonia in Wuhan, China. JAMA Intern Med. https://doi.org/10.1001/jamainternmed.2020.0994

2. (2020) MS International Federation Global COVID-19 advice for people with MS. Retrieved from: https://www.msif.org/wp-conte nt/uploads/2020/03/MSIF-Global-advice-on-COVID-19-for-peopl e-with-MS.docx.pdf. Accessed 27 May 2020

3. Italian Society of Neurology (SIN) and Italian MS Association (AISM) (2020) Indicazioni sulla gestione del paziente con SM nel corso di epidemia da COVID-19. Retrieved from: https://www. aism.it/sites/default/files/ComunicazioneGdSSINSM-Coronaviru s.pdf. Accessed 27 May 2020

4. Boeschoten RE, Braamse AMJ, Beekman ATF et al (2017) Prevalence of depression and anxiety in Multiple Sclerosis: a systematic review and meta-analysis. J Neurol Sci 372:331-341

5. Dahl OP, Stordal E, Lydersen S, Midgard R (2009) Anxiety and depression in multiple sclerosis. A comparative population-based study in Nord-Trøndelag County. Norway Mult Scler 15:14951501. https://doi.org/10.1177/1352458509351542

6. Feinstein A, O'Connor P, Gray T, Feinstein K (1999) The effects of anxiety on psychiatric morbidity in patients with multiple sclerosis. Mult Scler 5:323-326. https://doi.org/10.1177/1352458599 00500504

7. Korostil M, Feinstein A (2007) Anxiety disorders and their clinical correlates in multiple sclerosis patients. Mult Scler 13:67-72. https://doi.org/10.1177/1352458506071161

8. Beiske AG, Svensson E, Sandanger I et al (2008) Depression and anxiety amongst multiple sclerosis patients. Eur J Neurol 15:239245. https://doi.org/10.1111/j.1468-1331.2007.02041.x

9. Chwastiak L, Ehde DM, Gibbons LE et al (2002) Depressive symptoms and severity of illness in multiple sclerosis: epidemiologic study of a large community sample. Am J Psychiatry 159:1862-1868. https://doi.org/10.1176/appi.ajp.159.11.1862

10. Schiffer RB, Arnett P, Ben-Zacharia A et al (2005) The Goldman Consensus statement on depression in multiple sclerosis. Mult Scler J 11:328-337. https://doi.org/10.1191/1352458505ms 116 2oa

11. Siegert RJ, Abernethy DA (2005) Depression in multiple sclerosis: a review. J Neurol Neurosurg Psychiatry 76:469-475

12. Sadovnick AD, Remick RA, Allen J et al (1996) Depression and multiple sclerosis. Neurology 46:628-632. https://doi. org/10.1212/WNL.46.3.628

13. Giordano A, Granella F, Lugaresi A et al (2011) Anxiety and depression in multiple sclerosis patients around diagnosis. J Neurol Sci 307:86-91. https://doi.org/10.1016/j.jns.2011.05.008

14. Morrow SA, Drake A, Zivadinov R et al (2010) Predicting loss of employment over three years in multiple sclerosis: clinically meaningful cognitive decline. Clin Neuropsychol 24:1131-1145. https://doi.org/10.1080/13854046.2010.511272

15. Simmons RD (2010) Life issues in multiple sclerosis. Nat Rev Neurol 6:603-610

16. Smith MM, Arnett PA (2005) Factors related to employment status changes in individuals with multiple sclerosis. Mult Scler 11:602-609. https://doi.org/10.1191/1352458505ms1204oa

17. Thompson AJ, Banwell BL, Barkhof F et al (2018) Diagnosis of multiple sclerosis: 2017 revisions of the McDonald criteria. Lancet Neurol 17:162-173

18. Kurtzke JF (1983) Rating neurologic impairment in multiple sclerosis: an expanded disability status scale (EDSS). Neurology 33:1444-1452. https://doi.org/10.1212/wnl.33.11.1444

19. Amato MP, Portaccio E, Goretti B et al (2006) The Rao's Brief Repeatable Battery and Stroop Test: normative values with age, education and gender corrections in an Italian population. Mult Scler 12:787-793. https://doi.org/10.1177/1352458506070933

20. Van Schependom J, D'hooghe MB, Cleynhens K et al (2014) The Symbol Digit Modalities Test as sentinel test for cognitive impairment in multiple sclerosis. Eur J Neurol 21:1219-1225. https:// doi.org/10.1111/ene.12463(e71-e72)

21. Santangelo G, Sacco R, Siciliano M et al (2016) Anxiety in Multiple Sclerosis: psychometric properties of the State-Trait Anxiety Inventory. Acta Neurol Scand 134:458-466. https://doi. org/10.1111/ane.12564

22. Sacco R, Santangelo G, Stamenova S et al (2016) Psychometric properties and validity of Beck Depression Inventory II in multiple sclerosis. Eur J Neurol 23:744-750. https://doi.org/10.1111/ ene. 12932

23. Solari A, Filippini G, Mendozzi L et al (1999) Validation of Italian multiple sclerosis quality of life 54 questionnaire. J Neurol Neurosurg Psychiatry 67:158-162. https://doi.org/10.1136/ jnnp.67.2.158

24. Hochberg Y, Benjamini Y (1990) More powerful procedures for multiple significance testing. Stat Med 9:811-818. https://doi. org/10.1002/sim.4780090710

25. Naser Moghadasi A (2020) One aspect of coronavirus disease (COVID-19) outbreak in Iran high anxiety among MS patients. Mult Scler Relat Disord 41:102138. https://doi.org/10.1016/j. msard.2020.102138

26. Mazza C, Ricci E, Biondi S et al (2020) A Nationwide Survey of Psychological Distress among Italian People during the COVID19 pandemic: immediate psychological responses and associated factors. Int J Environ Res Public Health. https://doi.org/10.3390/ ijerph17093165

27. Perrin PC, McCabe OL, Everly GS, Links JM (2009) Preparing for an influenza pandemic: mental health considerations. Prehosp Disaster Med 24:223-230

28. Italian Health Ministry (2020) Retrieved: https://www.salute.gov. it/imgs/C_17_notizie_4767_0_file.pdf

29. Nazari F, Shaygannejad V, Mohammadi Sichani M et al (2020) Sexual dysfunction in women with multiple sclerosis: prevalence and impact on quality of life. BMC Urol. https://doi.org/10.1186/ s12894-020-0581-2

30. Tepavcevic DK, Kostic J, Basuroski ID et al (2008) The impact of sexual dysfunction on the quality of life measured by MSQoL-54 in patients with multiple sclerosis. Mult Scler 14:1131-1136. https ://doi.org/10.1177/1352458508093619

31. Blackmore DE, Hart SL, Albiani JJ, Mohr DC (2011) Improvements in partner support predict sexual satisfaction among individuals with multiple sclerosis. Rehabil Psychol 56:117-122. https ://doi.org/10.1037/a0023362

32. Henry A, Tourbah A, Camus G et al (2019) Anxiety and depression in patients with multiple sclerosis: the mediating effects of perceived social support. Mult Scler Relat Disord 27:46-51. https ://doi.org/10.1016/j.msard.2018.09.039

33. Mohr DC, Classen C, Barrera M (2004) The relationship between social support, depression and treatment for depression in people with multiple sclerosis. Psychol Med 34:533-541. https://doi. org/10.1017/S0033291703001235

34. Denis A, Callahan S, Bouvard M (2015) Evaluation of the French version of the multidimensional scale of perceived social support during the postpartum period. Matern Child Health J 19:12451251. https://doi.org/10.1007/s10995-014-1630-9

35. Laing CM, Cooper CL, Summers F et al (2019) The nature of anger in people with multiple sclerosis: a qualitative study. Psychol Heal. https://doi.org/10.1080/08870446.2019.1691726

36. Brooks SK, Webster RK, Smith LE et al (2020) The psychological impact of quarantine and how to reduce it: rapid review of the evidence. Lancet 395:912-920 
37. Solaro C, Trabucco E, Signori A et al (2016) Depressive symptoms correlate with disability and disease course in multiple sclerosis patients: an Italian multi-center study using the Beck
Depression Inventory. PLoS ONE. https://doi.org/10.1371/journ al.pone. 0160261 